\title{
Automated test-bed for long-range passive IR sensor
}

\author{
H. Madura, Z. Sikorski, H. Polakowski, M. Kastek, \\ Institute of Optoelectronics, Military University of Technology, \\ 2 Kaliskiego Str., 00-908 Warsaw
}

\begin{abstract}
The paper presents measuring possibilities of an automated test-bed used for measurements of parameters of longrange passive IR sensor. Scheme of configuration of the set up for measurements of both frequency and angular characteristics of sensors are shown. Exemplary results of measurements of passive IR sensor are also given.
\end{abstract}

\section{Introduction}

A long-range passive IR sensor, elaborated at the Institute of Optoelectronics MUT, forms passive barrier for detection of objects being in the range of about $200 \mathrm{~m}$ and the angles of view of $25^{\circ}$ and $1.5^{\circ}$, for vertical and horizontal directions, respectively. The sensor can detect an object with object-background temperature difference of the order of $0.2^{0} \mathrm{C}$. Testing the sensor, verification of design of its optical and electronic system, regulation of sensor sensitivity thresholds, and background-radiation compensation levels require application of automated test-bed. Because the passive IR sensor can detect even slight changes in IR radiation power, such a test-bed must fulfil very strict design requirements related to performance of optical and constructional elements of the test-bed [1].

\subsection{Test-bed functions}

The test-bed is used as a projector of radiative test simulating movement of standard object in the sensor's field of view. It is designed for testing of optical and electronic system of long-range passive IR sensors. The test-bed ensures possibility of evaluation of temperature resolution of the sensor, determination of amplification levels, and comparation thresholds. The frequency characteristics and characteristics vs. the angle of view can be also determined. Computer data registration, verification of correct alignment of optical system, and testing of decisive algorithm of the sensor are possible, too.

\subsection{Test-bed design}

A functional diagram of the test-bed is shown in Fig. 1. Main elements of the set-up are: the calibrated source of infrared radiation, digitally controlled rotary disk with exchangeable radiation testing-elements, IR collimator, precise and digitally controlled rotary table with a holder for fixing of the investigated sensor.

Measuring process is controlled by a software of a computer equipped with the measuring cards, oscilloscope cards and rotary table controller cards, as well as the cards of exchangeable radiation testing-elements. These testing-elements are placed close to the collimator focus. A blackbody source of infrared radiation, controlled thermoelectrically, enables to set the temperature of a radiation test in dependence on the required (positive or negative) contrast. The temperature of a testing-element 
can be changed with a resolution of $0.01 \mathrm{~K}$. Digitally-controlled rotary disc can be rotated with the angular velocities that correspond to the linear velocity range of the standard test of Polish standard PN-93/E-08390/26. According to this standard the test should be placed at the distance of $200 \mathrm{~m}$ to the sensor and its velocity should equal to $0.3-3 \mathrm{~m} / \mathrm{s}$. The precise rotary table ensures the sensor setting, in horizontal direction, with the accuracy of 0.050 . A control and data storage system employs an IBM PC computer with multi-channel measuring card.

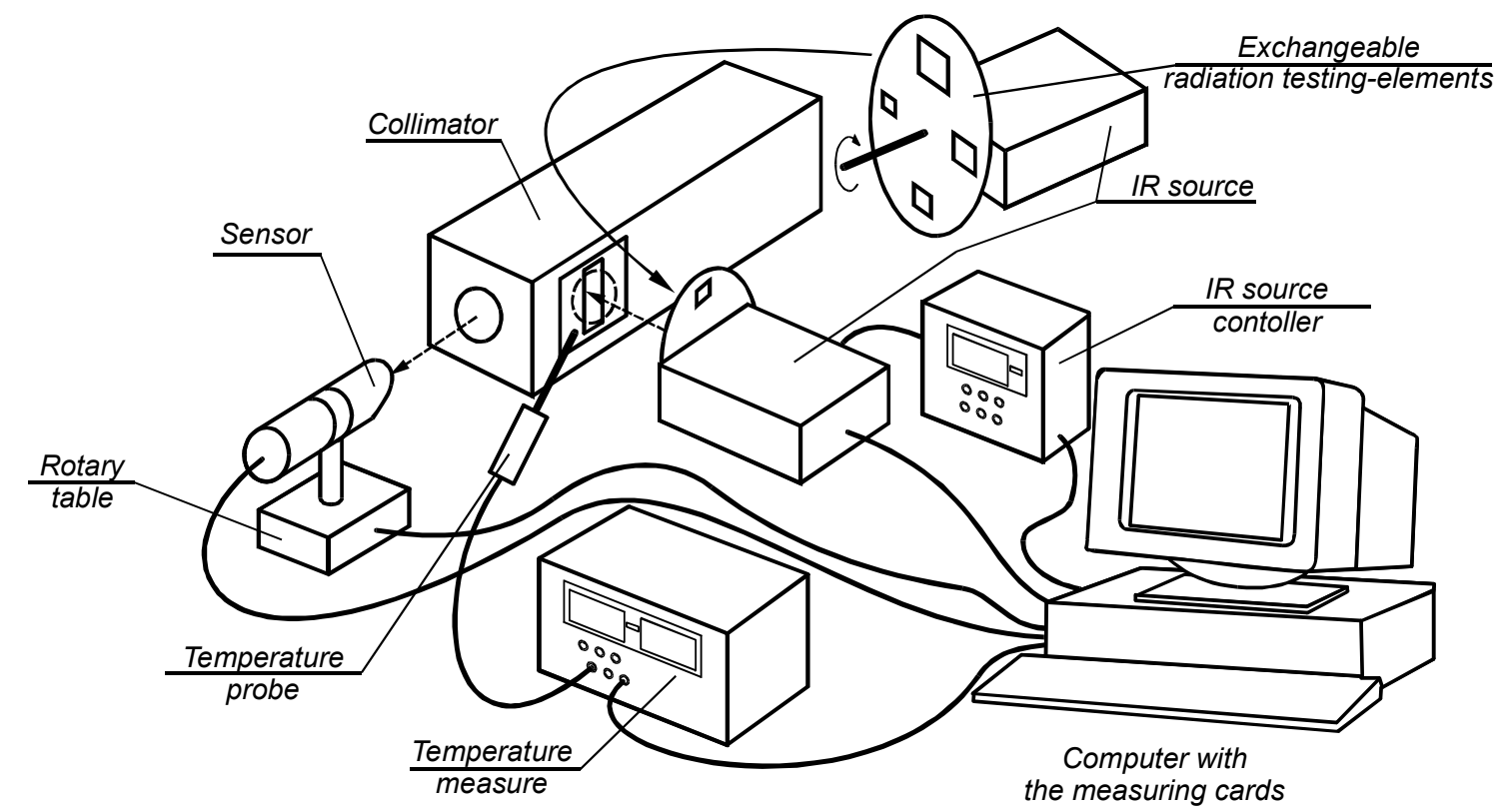

Fig. 1. A functional diagram of the test-bed.

A diaphragm, situated in front of the radiation testing-element, fulfils a role of background with ambient temperature. The temperature is measured by a contact method using measuring probe (70617E) with thermoelectric platinic sensor and temperature meter Solatron SJ-7061. Behind the diaphragm there is an exchangeable disk with holes. The holes simulate an object moving across the sensor's field of view, due to rotation of the disc with determined angular velocity. Frequency of rotation of the disc corresponds, after recalculation for linear velocity, to the velocity of moving object. Behind the disc there is also the blackbody source DCN 100 Infrarouge, the surface of which, observed through a hole in the disc, imitates the standard object, of the adequate dimensions and uniform distribution of temperatures, that moves in front of the detector.

A blackbody control unit allows setting the constant difference of temperatures (environment-testing-element) with accuracy of $0.1 \mathrm{~K}$. It enables setting of the difference between background temperature and temperature of standard objects that is required and constant during measurements.

\section{Frequency characteristics determination}

A measurement of signal amplitude changes is performed with a voltometer that is connected to the preamplifier output. Additionally connected oscilloscope allows observing the signals at the output of sensor's electronic system in the chosen measuring points. In order to determine frequency characteristic, the constant 
temperatures difference $\Delta \mathrm{T}$, between blackbody (object) and diaphragm (background), should be defined.

The measuring test-bed simulates a real system in which an object enters detector's field of view. Measurement consists in readout of a signal amplitude that changes vs. frequency of optical testing-element movement. Figure 2 shows two exemplary normalised frequency characteristics of the sensor for two differences of testing-element temperature and background temperature (for $\Delta \mathrm{T}_{1}=2 \mathrm{~K}$ and for $\left.\Delta \mathrm{T}_{1}=4 \mathrm{~K}\right)$.

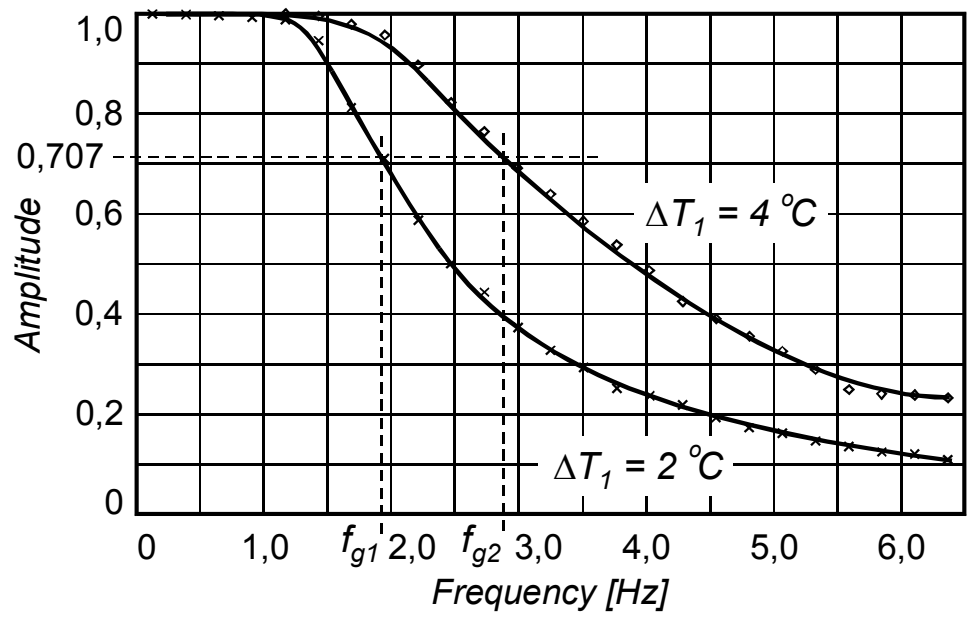

Fig. 2. Normalised frequency characteristics of the sensor for two differences of test temperature and background temperature (for $\Delta \mathrm{T} 1=2 \mathrm{~K}$ and for $\Delta \mathrm{T} 1=4 \mathrm{~K}$ ).

The dependence of limiting frequency of the sensor on a difference of testingelement temperature and background temperature $\Delta T$ (Fig. 3.) was obtained from frequency characteristics determined for testing-element temperature changed with background temperature.

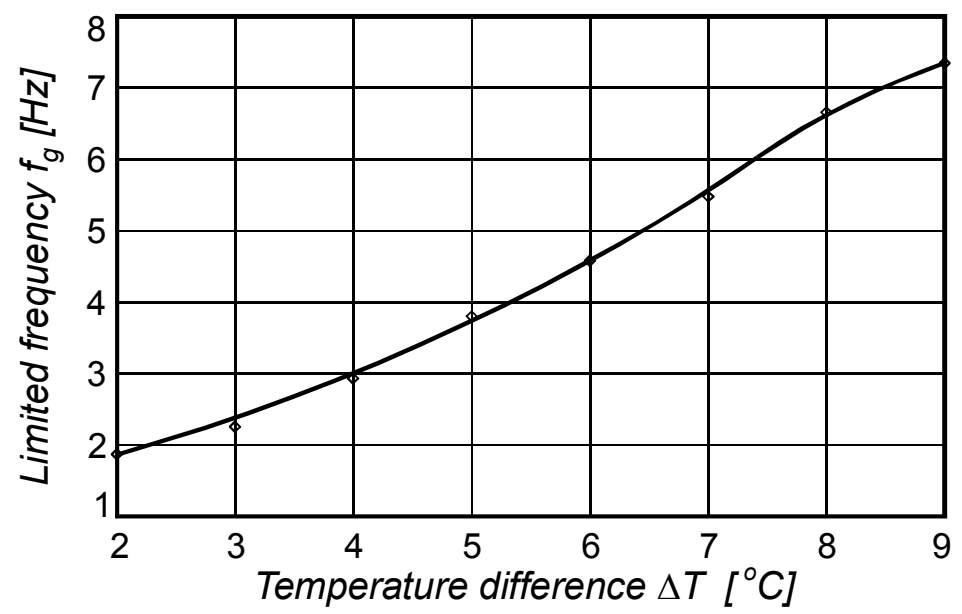

Fig. 3. Limiting frequency of the sensor vs. the difference of testing-element temperature and background temperature $(\Delta T)$.

Determination of frequency characteristics of infrared radiation allows determining of maximum velocity of the moving object that enables its detection for determined temperature difference of object-background. Simultaneously, the minimal difference $\Delta T_{\min }$ (temperature difference object-background) for which the simulated moving object causes alarm signal can be evaluated. Due to determined 
voltages, corresponding to $\Delta \mathrm{T}_{\min }$, the threshold values of a signal can be stated in the sensor's decisive system.

\section{Angular characteristic}

Angular characteristic of a sensor is determined in a configuration shown in Fig. 1. The investigated sensor is situated on a precise rotary table. An angular characteristic of the sensor is obtained by rotation of a table within the defined angular range (with the stated step) and signal amplitude readout by means of the computer measuring card. Readout of signal amplitude is synchronised with a table rotation. Additionally, oscilloscope is connected to the preamplifier output. It allows real time monitoring of the signals in electronic sensor's path. A precise rotary table is used for determination of angular characteristic with resolution of $0.05^{\circ}$.

Exemplary normalised angular characteristic of the sensor, for $\Delta \mathrm{T} 1=2 \mathrm{~K}$, is shown in Fig. 4.

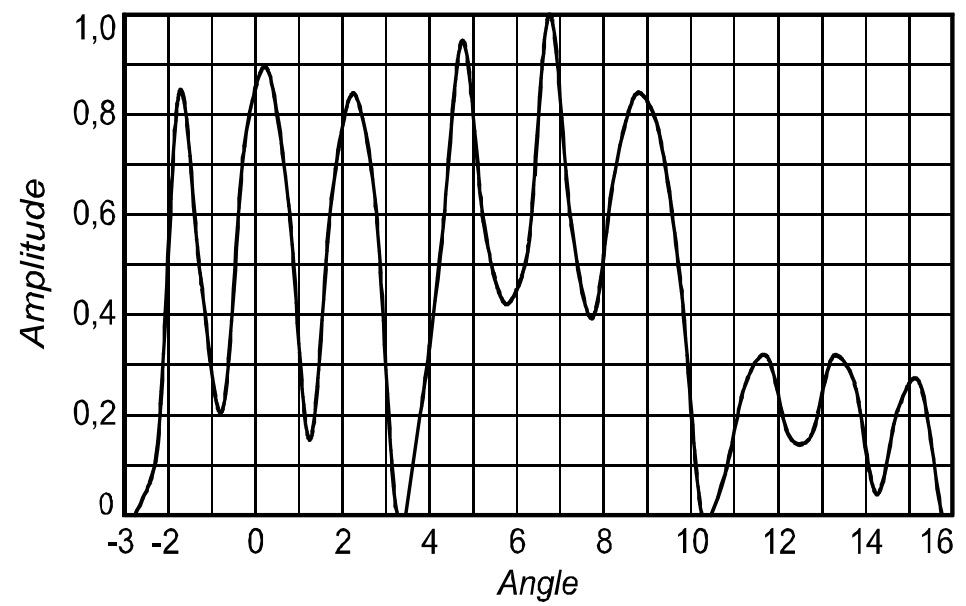

Fig. 4. Normalised angular characteristic of the sensor, for $\Delta \mathrm{T}_{1}=2 \mathrm{~K}$.

\section{Conclusions}

The described test bed allows testing and determination of decisive algorithm parameters as well as the systems for its accomplishment in IR sensors. Testing was performed against the velocity of an object moving through the protected area (set by the control of angular velocity of a rotary disk with radiation tests) and linear dimension of the object entering the protected area (set by replaceable disks with radiation tests of various hole dimensions). The sensor decisive thresholds can be established from evaluation of the minimum temperature differences $\Delta T_{\text {min }}$

\section{References}

[1] H. Polakowski, "Passive intelligent smart IR sensor of extended activity range used for protection systems of special objects", Report of grant No 148199/CT00/99, Warsaw, 2000 